\title{
Correlation between the Chebyshev Wavelets about the Derivatives Function
}

\author{
V.Ramalakshmi ${ }^{1}$, B.Ramesh Kumar $^{2}$ T.Balasubramanian ${ }^{3}$, S.Solaiappan ${ }^{4}$ \\ ${ }^{\text {I}}$ (Department of Mathematics, National Engineering College,Kovilpatti, TamilNadu, India) \\ ${ }^{2}$ (Department of Mathematics, Sree Sowdambika College of Engineering, Aruppukottai, TamilNadu, India) \\ 3(Department of Mathematics, Kamaraj College, Thoothukudi, TamilNadu, India) \\ 4(Department of Mathematics, Anna Univerity,Ramnad, TamilNadu, India)
}

\begin{abstract}
The aim of this paper is to derive the new correlation about the first and second kind of chebyshev wavelets and proposed to new results on the derivative function on chebyshev wavelets. It is most helpful for the optimal control analysis.
\end{abstract}

Keywords: Chebyshev Wavelet, Derivative of Chebyshev Wavelet, First and Second Chebyshev Wavelet.

\section{Introduction}

Many problems of mathematical physics, Engineering Science and applied science fields (Signal analysis, image processing, numerical analysis etc.,) can be stated in the form of integral and Differential equations. For this type of equations is simulation of the other mathematical problems such as PDF and ODE. Therefore, the study of the DE methods for solving is very useful application in Engineering and Pure and applied mathematics. Current years several methods are developed based on the orthogonal basic function with wavelet function. It has been very helpful for the approximate solution of integral and Differential equation $[1,8,10,11]$. In basic methods of wavelets are two important ways of improving the approximation solution. Develop the order of the wavelet family and the increasing the optimal control level of the wavelet. The basic idea of CWM is convert to the DE to a system of algebraic equation by the operational matrices of integral or derivative. Haar wavelets (Mirzaee, 2005), harmonic wavelets of successive approximation (Cattani and Kudreyko, 2010) Chebyshev polynomials is a traditional wavelet technique. The modified version which is called Chebyshev wavelets method. Chin.k[ 3] has proposed to convergence analysis of the fast approximation techniques in power electronic circuits. W.Jie [9] analyzed the steady state analysis of the power electronic wave transforms. Sohrabi.S [12] studied the comparatively of BPF and Abel's integral equation. Fathi.M.R [4] analyzed the time - invariant scaled operations on chebyshev wavelet. Hariharan and etc., $[5,6,7]$ have proposed the Haar Wavelet method for non-linear reaction arising in science and engineering. The main objectives of the wavelets are optimal control analysis of the signal process and etc., and it can be improved to analysis the convergence method. Chebyshev polynomials which are the eigen function of a Sturm-Liouville problem have many advantages [2]. In this paper we have proposed the connection between the about the shifted first and second chebyshev wavelet method and derive the derivative relation about the above function.

\section{Chebyshev Wavelets}

Wavelets constitute a family of functions constructed from dilation and translation of a single function $\psi(x)$ called the mother wavelet. When the dilation parameter " $\mathrm{a}$ " and the translation parameter " $\mathrm{b}$ " varies continuously we have the following family of continuous wavelets as $\psi_{a, b}(x)=|a|^{-1 / 2} \psi\left(\frac{x-b}{a}\right), a, b \in R, a \neq 0$. If the parameters a and $\mathrm{b}$ are discrete value, where $a=a_{0}^{-k}, b=n b_{0} a_{0}^{-k}, a_{0}>1, b_{0}>0$. The family of the discrete wavelet as follows: $\quad \psi_{k, n}(x)=|a|^{k / 2} \psi\left(a_{0}^{k} x-n b_{0}\right), k, n \in R$, where $\quad \psi_{k, n} \in L^{2}(R)$. Suppose that, here $a_{0}=2$ and $b=1$, the family of discrete wavelet forms as an orthonormal basis. The weight functions of the chebyshev wavelet denoted by $\mathrm{w}(\mathrm{x})$ and it's dilated and translated as $w_{n}(x)=w\left(2^{k} x-2 n+1\right)$ Chebyshev polynomial most helpful for the numerical analysis.

\subsection{First Chebyshev Wavelets:}

Four arguments are involving this kinds of wavelet, the family of wavelet is $\psi_{k, n}^{1}(x)=\psi^{1}(k, n, m, x)$, where $n=1,2,3 \ldots .2^{k}, k \in R^{+}, \mathrm{m}$ - denotes the degree of Chebyshev Polynomial, $\mathrm{n}$ arguments, $\mathrm{k}$ can assume any positive integer, $\mathrm{x}$ is the normalized time. It is defined on the interval $[0,1]$ by 
$\psi^{1}{ }_{m, n}(x)=\left\{\begin{array}{c}2^{(k+1) / 2} \bar{T}_{m}\left(2^{k+1} x-2 n-1\right), \frac{n}{2^{k}} \leq x<\frac{n+1}{2^{k}} \\ \text { otherwise }\end{array}\right\}$ where $\bar{T}_{m}(x)=\sqrt{2 / \pi} T_{m}(x), \mathrm{M}=0,1,2 \ldots \mathrm{M}$ and $\mathrm{n}=0,1, \ldots 2^{\mathrm{k}}$.

\subsection{Second Chebyshev Wavelets:}

Four arguments are involving this kinds of wavelet, the family of wavelet is. $\psi_{k, n}^{2}(x)=\psi^{2}(k, n, m, x)$, n arguments, $\mathrm{k}$ can assume any positive integer, $\mathrm{x}$ is the normalized time. It is defined on the interval $[0,1]$ by $\psi_{n, m}^{2}(x)=\left\{2^{k / 2} \bar{V}_{m}\left(2^{k+1} x-2 n-1\right), \frac{n}{2^{k-1}} \leq x \leq \frac{n+1}{2^{k-1}}\right\}, \quad---(2)$ where $\quad \bar{V}_{m}(x)=\sqrt{2 / \pi} V_{m}(x), \quad \mathrm{M}=0,1,2 \ldots \mathrm{M}$, $\mathrm{n}=0,1,2 \ldots .2^{(\mathrm{k}-1)}-1$. A function $\mathrm{f}(\mathrm{x})$ defined over $[0,1]$ can be expanded interms of $\left(\psi_{n, m}\right.$ either $\psi_{p}^{1}(x)$ or $\psi_{p}^{2}(x)$ . Here $f(x)=\sum_{n=0}^{\infty} \sum_{m=0}^{\infty} C_{n, m} \psi_{n, m}$, where $C_{n, m}=\left(f(x), \psi_{n, m}(x)\right)$----- (3), If the infinite series (3) is truncated and it can written as $\quad f(x)=\sum_{n=0}^{2^{k-1}} \sum_{m=0}^{M-1} C_{n, m} \psi_{n, m}$

$C=\left[c_{00}, c_{01}, c_{02,} \ldots c_{0 M}, \ldots c_{\left(2^{k}-1\right) M}, \ldots c_{\left(2^{k}-1\right) 0}, c_{\left(2^{k}-1\right) 1} \ldots c_{\left(2^{k}-1\right) M}\right]^{T} \quad$ and $\psi(x)=\left[\psi_{00}, \psi_{01}, \psi_{02}, \ldots \psi_{0 M}, \ldots \psi_{\left(2^{k}-1\right) M}, \ldots \psi_{\left(2^{k}-1\right) 0}, \psi_{\left(2^{k}-1\right) !} \ldots \psi_{\left(2^{k}-1\right) M}\right]^{T} \quad------\quad(5)$ then $C$ and $\psi(x)$ are $2^{k}(M+1) \times 1$ matrices.

III. Correlation Between The Function of $\psi_{n, m}^{1}$ and $\psi_{n, m}^{2}$

For $\psi_{n, m}^{2}-\psi_{n, m-2}^{2}=\frac{2 \sqrt{2}}{c_{m}} \psi_{n m}^{1}(x)$----- (6), where $c_{m}=\left\{\begin{array}{ll}\sqrt{2} & m=0 \\ 2 & m=1,2 \ldots\end{array}\right\}$----- (7)

From the relation about the $U_{m}$ and $T_{m}$ is $V_{m}(x)-V_{m-2}(x)=2 T_{m}(x), \mathrm{m}=2,3 \ldots \ldots$ we have

$$
\begin{aligned}
& \psi_{n m}^{2}=\sqrt{2 / \pi} 2^{k / 2}\left(2 T_{m}+V_{m-2}\right)\left(2^{k+1}-2 n+1\right) \\
& =\sqrt{2 / \pi} 2^{k / 2} 2 T_{m}\left(2^{k+1}-2 n+1\right)+\sqrt{2 / \pi} 2^{k / 2} V_{m-2}\left(2^{k+1}-2 n+1\right) \\
& \psi_{n, m}^{2}=\psi_{n, m-2}^{2}+2^{k / 2} \sqrt{2 / \pi} 2 T_{m}\left(2^{k+1}-2 n+1\right), \mathrm{m}=2,3 \ldots
\end{aligned}
$$

\subsection{Correlation about the Matric Derivative of $\psi_{n, m}^{1}$ and $\psi_{n, m}^{2}$}

Lemma: 1 Let $\psi^{1}(x)$ be the chebyshev wavelets defined by

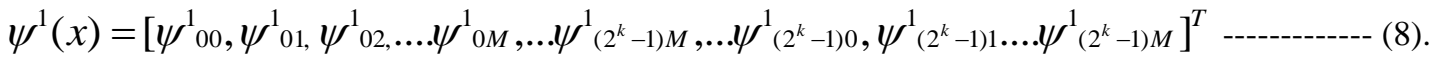

This derivative of this vector $\psi^{1}(x)$ can be expressed by $\frac{d \psi^{1}}{d x}=D_{\psi^{\prime}} \psi^{1}(x)$, where $\mathrm{D}$ is the $2^{k}(M+1)$ operational matrix defined as follows: $D_{\psi^{\prime}}=\left[\begin{array}{ccccc}R & 0 & \ldots . & \ldots & 0 \\ 0 & R & \ldots & \ldots . & 0 \\ \ldots & \ldots & \ldots & \ldots & \ldots \\ \ldots & \ldots & \ldots & \ldots & \ldots \\ 0 & 0 & \ldots . & \ldots & R\end{array}\right]$,

Here $\mathrm{T}$ is $(M \times 1) \times(M \times 1)$ matrix and (p,t) the element is defined as follow:

$R_{p, t}=\left\{2^{k+1} m \quad p=2,3 \ldots .(M+1), t=1.2 \ldots . r-1\right\}$

Lemma:2 Let $\psi^{2}(x)$ be the chebyshev wavelets defined by

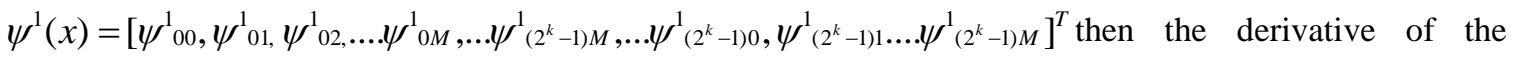
$\psi^{2}(x)$ can be expressed by $\frac{d \psi^{2}}{d x}=D_{\psi^{2}} \psi^{2}(x)$, where $D_{\psi^{2}}(x)$ is the $2^{k-1}(M+1)$ 
$D_{\psi 2}=\left[\begin{array}{ccccc}Q & 0 & \ldots \ldots & \ldots . & 0 \\ 0 & Q & \ldots . & \ldots . & 0 \\ \ldots & \ldots & \ldots & \ldots . & \ldots \\ \ldots & \ldots & \ldots & \ldots & \ldots \\ 0 & 0 & \ldots & \ldots & Q\end{array}\right]$-------- (10),

In which $Q$ is $\left(\begin{array}{lllll}M & x\end{array}\right) \times\left(\begin{array}{lll}M & 1\end{array}\right)$ matrix and its $(p, t)$ the element is defined as follows: $Q_{p, t}=\left\{\begin{array}{cc}2^{k+1} t & p=2,3 \ldots . .(M+1) \\ 0 & \text { Otherwisw }\end{array}\right.$

Proof: Using the shifted Second Chebyshev polynomial to the interval $[0,1]$, the pth element of vector $\psi^{2}(x)$ can $\quad$ be $\quad$ written as $\psi_{p}^{2}(x)=\left\{2^{k / 2} \sqrt{2 / \pi} \overline{V_{m}}\left(2^{k} x-n\right) \quad x \in\left[\frac{n}{2^{k-1}}, \frac{n-1}{2^{k-1}}\right]\right\}$-------(12), where

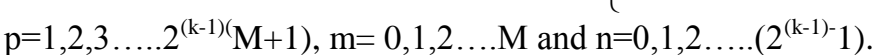

Differentiating the equation (12) with respect to $\mathrm{x}$, we have

$\frac{d \psi_{p}^{2}(x)}{d x}=\left\{2^{k / 2} \sqrt{2 / \pi} \overline{V_{m}}\left(2^{k} x-n\right) \quad x \in\left[\frac{n}{2^{k-1}}, \frac{n-1}{2^{k-1}}\right]\right\}$

i.e $\psi_{j}^{2}(x), \mathrm{j}=\mathrm{n}(\mathrm{M}+1), \mathrm{n}(\mathrm{M}+2) \ldots \ldots(\mathrm{n}+1)(\mathrm{M}+1)$. Therefore, in general we expanse the second chebyshev wavelet $\frac{d \psi_{p}^{2}}{d t}=\sum_{i=n(M+1)+1}^{(n+1)(M+1)} a_{j} \psi_{j}^{2}$

This implies that the operational matrix $D_{\psi^{2}}$ moreover, we have $\frac{d \psi_{p}^{2}}{d t}=0$, consequently the row of matrix (11) is also zero.

First derivative of the shifted second chebyshev polynomial is $\bar{V}_{m}^{,}=\sum_{k=0}^{m-1} 4(k+1) \bar{V}_{m}(x) \quad m=1,2, \ldots m+k($ odd $)$

$$
\begin{aligned}
\frac{d \psi_{p}^{2}(x)}{d x} & =2^{k / 2} \sqrt{2 / \pi} 2^{k} \sum_{i=0}^{m-1} 4(i+1) \bar{V}_{i}\left(2^{k-1}(x)-n\right) \quad(i+m) \\
& =2^{k+1} \sum_{t=1}^{p-1} p \psi_{n(M+1)+s}^{2}(x) \quad(p+t)---------
\end{aligned}
$$

So, we consider $Q_{p, t}=\left\{\begin{array}{ll}2^{k+1} t & p=2,3 \ldots . .(M+1), t=1,2 \ldots p-1 \\ 0 & \text { otherwise }\end{array}\right\}$----- (16)

Hence the required result.

\section{Correlation between New Derivatives of The Function $\psi_{n, m}^{1}$ and $\psi_{n, m}^{2}$}

Lemma: 3 The derivative of $\psi_{p}^{1}$ and $\psi_{p}^{2}$ is formulated as $\frac{d \psi_{p}^{1}(x)}{d x}=2^{k+1} m \sum_{t=1}^{p-1} \psi_{n(M+1)+p}^{2}---(17)$

Proof: Using the first shifted chebyshev polynomial in [0,1], $\psi_{n, m}^{1}(x)$ can be written as

$\psi_{n, m}^{1}(x)=\left\{\begin{array}{ll}2^{(k+1) / 2} \bar{T}_{m}\left(2^{k+1}(x)-2 n-1\right. & \frac{n}{2^{k}} \leq x \leq \frac{n+1}{2^{k}} \\ 0 & \text { otherwise }\end{array}\right\}$

Then differentiation with respect to $\mathrm{x}$, we obtained

$$
\begin{aligned}
& \frac{d \psi_{p}^{1}(x)}{d x}=2^{k} \sum_{t=1}^{p-1} 4 m \sqrt{\frac{c_{p-1}}{c_{t-1}}} \psi_{n(M+1)+s}^{1}(x) \quad p=2,3 \ldots . .(M+1), t=1,2 \ldots . p-1 \\
& =2^{k+2} m \sqrt{\frac{c_{p-1}}{c_{t-1}}} \sum_{t=1}^{p-1} \psi_{n(M+1)+p}^{1}(x) \quad(p+t) i s \text { odd } \\
& \frac{d \psi_{p}^{1}(x)}{d x}=\left(2^{k+1} m\right) 2 \sqrt{\frac{c_{p-1}}{c_{t-1}}} \sum_{t=1}^{p-1} \psi_{n(M+1)+p}^{1}(x) \quad p=2,3 \ldots .(M+1)^{--------(18)}
\end{aligned}
$$


Since $\psi_{p-1}^{1}(x)=2 / \sqrt{\pi}$ then $\frac{d \psi_{p}^{1}(x)}{d x}=2^{k+1} m \sqrt{2} \psi_{p-1}^{1}$

i.e) $\frac{d \psi_{p}^{1}(x)}{d x}=2^{k+1} m \psi_{p-1}^{2}(x)$,

Finally, if $\frac{c_{p-1}}{c_{t-1}}=1$. Then the equation (18) becomes $\frac{d \psi_{p}^{1}(x)}{d x}=2^{k+1} m \sum_{t=1}^{p-1} \psi_{n(M+1)+p}^{2}$.

Lemma: 4 The derivative of $\psi_{p}^{2}(x)$ interms of $\psi_{p}^{1}(x)$ and $\psi_{p-1}^{2}(x)$ is formulated as

$$
\frac{d \psi_{p}^{2}(x)}{d x}=\left(2^{k+1} t\right)\left[\sum_{t=1}^{p-1} \frac{2 \sqrt{2}}{c_{s-1}} \psi_{n(M+1)+p}^{t}(x)+\sum_{t=1}^{p-1} \psi_{n(M+1)+p-2}^{2}(x) \quad p=2,3 \ldots(M+1), t=1,23 \ldots p-1\right]--
$$

Proof: From the lemma (2) $\frac{d \psi_{p}^{2}(x)}{d x}=2^{k+1} t \sum_{t=1}^{p-1} \psi_{n(M+1)+p}^{2}(x) \quad p+t=o d d$

Using the equation (20) in $\psi_{n m}^{1}$ and $\psi_{n m}^{2}$ which is the required result

Lemma: 5 The derivative of $\psi_{p}^{2}(x)$ interms of the second derivative of $\psi_{p}^{1}(x)$ is given by the formula

$$
\frac{d \psi_{p}^{2}(x)}{d x}=\frac{1}{2^{k+1} m} \sum_{t=1}^{p} \psi_{n(M+1)+p+1}^{1}(x) \quad(p+t) \text { is anodd }
$$

Proof: From the lemma (3) $\frac{d \psi_{p}^{1}(x)}{d x}=\left(2^{k+1} m\right) \sum_{t=1}^{p-1} \psi_{n(M+1)+p}^{2}(x)$

Differentiation above equation to obtain $\frac{d^{2} \psi_{p}^{1}(x)}{(d x)^{2}}=\left(2^{k+1} m\right) \sum_{t=1}^{p-1} \frac{d \psi_{n(M+1)+p}^{2}(x)}{d x}$

Divide both sides $\left(2^{k+1} m\right)$, we get $\frac{1}{2^{k+1} m} \frac{d^{2} \psi_{p}^{1}(x)}{(d x)^{2}}=\sum_{t=1}^{p-1} \frac{d \psi_{n(M+1)+p}^{2}(x)}{d x}$, here $\mathrm{r}=\mathrm{n}(\mathrm{M}+1)+\mathrm{s}+1$

Therefore, $\frac{d^{2} \psi_{p}^{1}(x)}{(d x)^{2}}=\frac{1}{2^{k+1} m} \sum_{t=1}^{p} \psi_{n(M+1)+p+1}^{1}(x)$, where $\mathrm{p}=3,4 \ldots .(\mathrm{M}+1), \mathrm{t}=1,2, \ldots \ldots(\mathrm{p}-1)$

Hence the required result

\section{Conclusion}

In this paper, we derived the correlation between first and second kind of chebyshev wavelet method and to derive the derivative about the above function. It is used to derive the analytical solution of the optimal control analysis. We conclude that the proposed algorithm is most helpful for the chebyshev wavelets and their operational matrices of derivatives.

\section{References}

[1] A. Alipanah and M. Dehghan, Numerical solution of the nonlinear Fredholm integral equations by positive definite functions, Applied Mathematics and Computation, 190(2), 2007, 1754-1761

[2] E.Babolian and F.Fattah Zadeh, Numerical Solution of differential equation by using Chebyshev Wavelet Oprational Matrix of Integration, Applied Mathematics and Computation, 188, 2007, 417-426

[3] K.T. Chi, A wavelet approach to fast approximation of steady-state waveforms of power electronics circuits, Int. J. Circ. Theor. Appl. 31, 2003, 591-610

[4] M.R.Fatihi and M.Smavat., State Analysis and optimal control of Linear Time-Invariant Scaled Systems Using the Chebyshev Wavelets, Contemporary Engineering Sciences, .20(5), 2012, 91-105,

[5] G.Hariharan and K.Kannan, Haar wavelet in estimating depth profile of soil temperature, Appl.Math.Comput., 21, 2009, 119-225

[6] G.Hariharan and K.Kannan, A Comparison of Haar Wavelet and Adomain decomposition method for solving one-dimensional reaction diffusion equation, Int.J.Appl.Mat.Comput. 2(1), 2010, 50-61

[7] G.Hariharan and K.Kannan, Haar Wavelet Method for solving nonlinear parabolic equations, J.Mat.Comput, 211, 2009, 284-292

[8] C. Hsiao, Hybrid function method for solving Fredholm and Volterra integral equations of the second kind, Journal of Computational and Applied Mathematics, 230(1), 2009, 59-68,

[9] W.Jie, and M.Liu., Application of Wavelet Transform to steady-state Approximation of power electronic Waveforms, Department of Electronic, Hong Kong Polytechnic University, 2003.

[10] M. Razzaghi and Y. Ordokhani, Solution of nonlinear Volterra-Hammerstein integral equations via rationalized Haar functions, Mathematical Problems in Engineering, 7(2), 2001, 205-219,

[11] M. Razzaghi and Y. Ordokhani, A rationalized Haar functions method for nonlinear Fredholm-Hammerstein integral equations, International Journal of Computer Mathematics, 79(3), 2002.333-343,

[12] S.Sohrabi., Comparison Chebyshev Wavelets Method with BPFs Method for Solving Abel's Integral Equation, In sharms Engineering Journal, 2, 2011, 